\title{
Reduced-Intensity Delayed Intensification in Standard-Risk Pediatric Acute Lymphoblastic Leukemia Defined by Undetectable Minimal Residual Disease: Results of an International Randomized Trial (AIEOP-BFM ALL 2000)
}

Schrappe, Martin ; Bleckmann, Kirsten ; Zimmermann, Martin ; Biondi, Andrea ; Möricke, Anja ; Locatelli, Franco ; Cario, Gunnar ; Rizzari, Carmelo ; Attarbaschi, Andishe ; Valsecchi, Maria Grazia ; Bartram, Claus R ; Barisone, Elena ; Niggli, Felix ; Niemeyer, Charlotte ; Testi, Anna Maria ; Mann, Georg ; Ziino, Ottavio ; Schäfer, Beat ; Panzer-Grümayer, Renate ; Beier, Rita ; Parasole, Rosanna ; Göhring, Gudrun ; Ludwig, Wolf-Dieter ; Casale, Fiorina ; Schlegel, Paul-Gerhardt ; Basso, Giuseppe ; Conter, Valentino

\begin{abstract}
Purpose Delayed intensification (DI) is an integral part of treatment of childhood acute lymphoblastic leukemia (ALL), but it is associated with relevant toxicity. Therefore, standard-risk patients of trial AIEOP-BFM ALL 2000 (Combination Chemotherapy Based on Risk of Relapse in Treating Young Patients With ALL) were investigated with the specific aim to reduce treatment intensity. Patients and Methods Between July 2000 and July 2006, 1,164 patients (1 to 17 years of age) with standard-risk ALL (defined as the absence of high-risk cytogenetics and undetectable minimal residual disease on days 33 and 78) were randomly assigned to either experimental reduced-intensity DI (protocol III; P-III) or standard DI (protocol II; P-II). Cumulative drug doses of P-III were reduced by $30 \%$ for dexamethasone and $50 \%$ for vincristine, doxorubicin, and cyclophosphamide, which shortened the treatment duration from 49 to 29 days. The study aimed at noninferiority of reduced-intensity P-III; analyses were performed according to treatment given. Results For P-III and P-II, respectively, the 8-year rate of disease-free survival $( \pm \mathrm{SE}$ ) was $89.2 \pm 1.3 \%$ and $92.3 \pm 1.2 \%(\mathrm{P}=.04)$; cumulative incidence of relapse, $8.7 \pm$ $1.2 \%$ and $6.4 \pm 1.1 \%(\mathrm{P}=.09)$; and overall survival, $96.1 \pm 0.8 \%$ and $98.0 \pm 0.6 \%(\mathrm{P}=.06)$. Patients with ETV6-RUNX1-positive ALL and patients 1 to 6 years of age performed equally well in both arms. The incidence of death during remission was comparable, which indicates equivalent toxicity. The 8-year cumulative incidence rate of secondary malignancies was $1.3 \pm 0.5 \%$ and $0.6 \pm 0.4 \%$ for P-III and P-II, respectively $(\mathrm{P}=.37$ ). Conclusion Although the criteria used for the standard-risk definition in this trial identified patients with exceptionally good prognosis, reduction of chemotherapy was not successful mainly because of an increased rate of relapse. The data suggest that treatment reduction is feasible in specific subgroups, which underlines the biologic heterogeneity of this cohort selected according to treatment response.
\end{abstract}

DOI: https://doi.org/10.1200/JCO.2017.74.4946

Posted at the Zurich Open Repository and Archive, University of Zurich ZORA URL: https://doi.org/10.5167/uzh-147297

Journal Article

Published Version 
Originally published at:

Schrappe, Martin; Bleckmann, Kirsten; Zimmermann, Martin; Biondi, Andrea; Möricke, Anja; Locatelli, Franco; Cario, Gunnar; Rizzari, Carmelo; Attarbaschi, Andishe; Valsecchi, Maria Grazia; Bartram, Claus R; Barisone, Elena; Niggli, Felix; Niemeyer, Charlotte; Testi, Anna Maria; Mann, Georg; Ziino, Ottavio; Schäfer, Beat; Panzer-Grümayer, Renate; Beier, Rita; Parasole, Rosanna; Göhring, Gudrun; Ludwig, Wolf-Dieter; Casale, Fiorina; Schlegel, Paul-Gerhardt; Basso, Giuseppe; Conter, Valentino (2018). Reduced-Intensity Delayed Intensification in Standard-Risk Pediatric Acute Lymphoblastic Leukemia Defined by Undetectable Minimal Residual Disease: Results of an International Randomized Trial (AIEOPBFM ALL 2000). Journal of Clinical Oncology, 36(3):244-253.

DOI: https://doi.org/10.1200/JCO.2017.74.4946 
Author affiliations and support information (if applicable) appear at the end of this article.

Published at jco.org on November 17, 2017.

M.S., K.B., and M.Z. contributed equally to this work.

G.B. and V.C. contributed equally to this work

Clinical trial information: NCT00430118 and NCT00613457

Corresponding author: Martin Schrappe, MD, PhD, Department of Pediatrics, University of Kiel and University Medical Center Schleswig-Holstein, Campus Kiel, Schwanenweg 20, 24105 Kiel, Germany; e-mail: m.schrappe@pediatrics.uni-kiel.de.

C 2017 by American Society of Clinical Oncology

0732-183X/18/3603w-244w/\$20.00

\section{Reduced-Intensity Delayed Intensification in Standard-Risk Pediatric Acute Lymphoblastic Leukemia Defined by Undetectable Minimal Residual Disease: Results of an International Randomized Trial (AIEOP-BFM ALL 2000)}

Martin Schrappe, Kirsten Bleckmann, Martin Zimmermann, Andrea Biondi, Anja Möricke, Franco Locatelli, Gunnar Cario, Carmelo Rizzari, Andishe Attarbaschi, Maria Grazia Valsecchi, Claus R. Bartram, Elena Barisone, Felix Niggli, Charlotte Niemeyer, Anna Maria Testi, Georg Mann, Ottavio Ziino, Beat Schäfer, Renate PanzerGrümayer, Rita Beier, Rosanna Parasole, Gudrun Göhring, Wolf-Dieter Ludwig, Fiorina Casale, Paul-Gerhardt Schlegel, Giuseppe Basso, and Valentino Conter

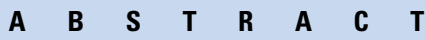

\section{Purpose}

Delayed intensification (DI) is an integral part of treatment of childhood acute lymphoblastic leukemia (ALL), but it is associated with relevant toxicity. Therefore, standard-risk patients of trial AIEOP-BFM ALL 2000 (Combination Chemotherapy Based on Risk of Relapse in Treating Young Patients With ALL) were investigated with the specific aim to reduce treatment intensity.

\section{Patients and Methods}

Between July 2000 and July 2006, 1,164 patients (1 to 17 years of age) with standard-risk ALL (defined as the absence of high-risk cytogenetics and undetectable minimal residual disease on days 33 and 78) were randomly assigned to either experimental reduced-intensity DI (protocol III; P-III) or standard DI (protocol II; P-II). Cumulative drug doses of P-III were reduced by $30 \%$ for dexamethasone and 50\% for vincristine, doxorubicin, and cyclophosphamide, which shortened the treatment duration from 49 to 29 days. The study aimed at noninferiority of reduced-intensity P-III; analyses were performed according to treatment given.

\section{Results}

For P-III and P-II, respectively, the 8-year rate of disease-free survival ( \pm SE) was $89.2 \pm 1.3 \%$ and $92.3 \pm 1.2 \%(P=.04)$; cumulative incidence of relapse, $8.7 \pm 1.2 \%$ and $6.4 \pm 1.1 \%(P=.09)$; and overall survival, $96.1 \pm 0.8 \%$ and $98.0 \pm 0.6 \%(P=.06)$. Patients with ETV6-RUNX1-positive ALL and patients 1 to 6 years of age performed equally well in both arms. The incidence of death during remission was comparable, which indicates equivalent toxicity. The 8-year cumulative incidence rate of secondary malignancies was $1.3 \pm 0.5 \%$ and $0.6 \pm 0.4 \%$ for $\mathrm{P}-\mathrm{II}$ and $\mathrm{P}-\mathrm{II}$, respectively $(P=.37)$.

\section{Conclusion}

Although the criteria used for the standard-risk definition in this trial identified patients with exceptionally good prognosis, reduction of chemotherapy was not successful mainly because of an increased rate of relapse. The data suggest that treatment reduction is feasible in specific subgroups, which underlines the biologic heterogeneity of this cohort selected according to treatment response.

\section{J Clin Oncol 36:244-253. (c) 2017 by American Society of Clinical Oncology}

\section{INTRODUCTION}

Over the past decades, the prognosis for children and adolescents with acute lymphoblastic leukemia (ALL) has improved considerably. Advances were mainly accomplished through refinement of biologic characterization, risk group assignment, and risk-stratified treatment. ${ }^{1-4}$ The assessment of minimal residual disease (MRD) has introduced unsurpassed precision in the differentiation between patients with a high risk of relapse and those with a low risk. ${ }^{5-7}$ Prospectively developed in the 1990s, MRD measured by immunoglobulin/ $\mathrm{T}$-cell receptor gene rearrangement polymerase chain reaction (PCR-MRD) was implemented for risk stratification in the trial, Combination Chemotherapy Based on Risk of Relapse in Treating Young Patients with Acute Lymphoblastic Leukemia (AIEOP-BFM ALL 2000). ${ }^{2,3,6}$
DOI: https://doi.org/10.1200/JCO 2017.74.4946 


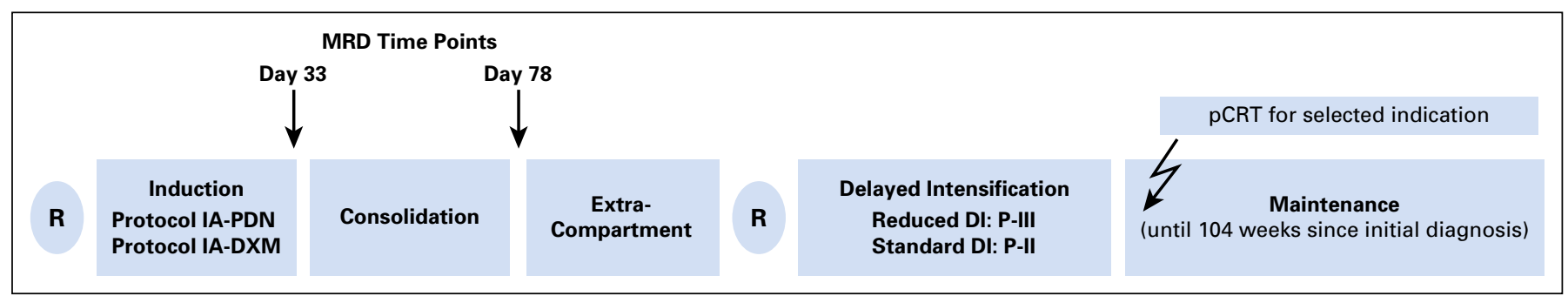

Fig 1. Treatment outline of the standard-risk arm of the study AIEOP-BFM ALL 2000. DI, delayed intensification; DXM, dexamethasone; MRD, minimal residual disease; pCRT, preventive cranial radiotherapy; Protocol IA-DXM, protocol I-A (induction regimen) with dexamethasone; Protocol IA-PDN, protocol I-A with prednisone; P-II, protocol II; P-III, protocol III; R, randomization.

With increasing survival rates, therapy-related morbidity and mortality as well as long-term sequelae have increasingly moved into focus. ${ }^{8,9}$ Especially for patient groups with the most favorable prognosis, several leukemia study groups have strived for a reduction of treatment burden without jeopardizing outcome. ${ }^{10-14}$

In former trials, the ALL-Berlin-Frankfurt-Münster (BFM) study group demonstrated the importance of delayed intensification in the treatment of patients with low-risk ALL. The reintensification element protocol II (P-II) significantly improved the outcome of high-risk patients in ALL-BFM $76 .{ }^{15}$ The reintensification element (protocol III [P-III]) also was implemented for low-risk patients in ALL-BFM 79. ${ }^{16,17}$ ALL-BFM 83 focused again on reduction of treatment burden in low-risk patients by testing treatment with and without P-III, which yielded results in favor of reinduction (10-year probable event-free survival [pEFS] rate, $81 \pm 5 \% v 56 \pm 6 \%)^{16,18}$
In the era of improved risk assignment, the question of the adequate chemotherapeutic intensity needed to ensure consistently low relapse rates was again addressed in a cooperative prospective trial jointly conducted by two large leukemia study groupsAssociazione Italiana di Ematologia e Oncologia Pediatrica (AIEOP) and Berlin-Frankfurt-Münster (BFM)—in AIEOP-BFM ALL 2000. The randomized trial compared standard delayed intensification (P-II) with a reduced-intensity regimen (P-III) in a cohort of standardrisk (SR) patients with favorable treatment response defined by PCRMRD. Herein, we report the results of this trial.

\section{PATIENTS AND METHODS}

\section{Study Design}

Patients 1 to 17 years of age with ALL in one of the participating centers in Italy, Germany, Austria, and Switzerland were registered in the

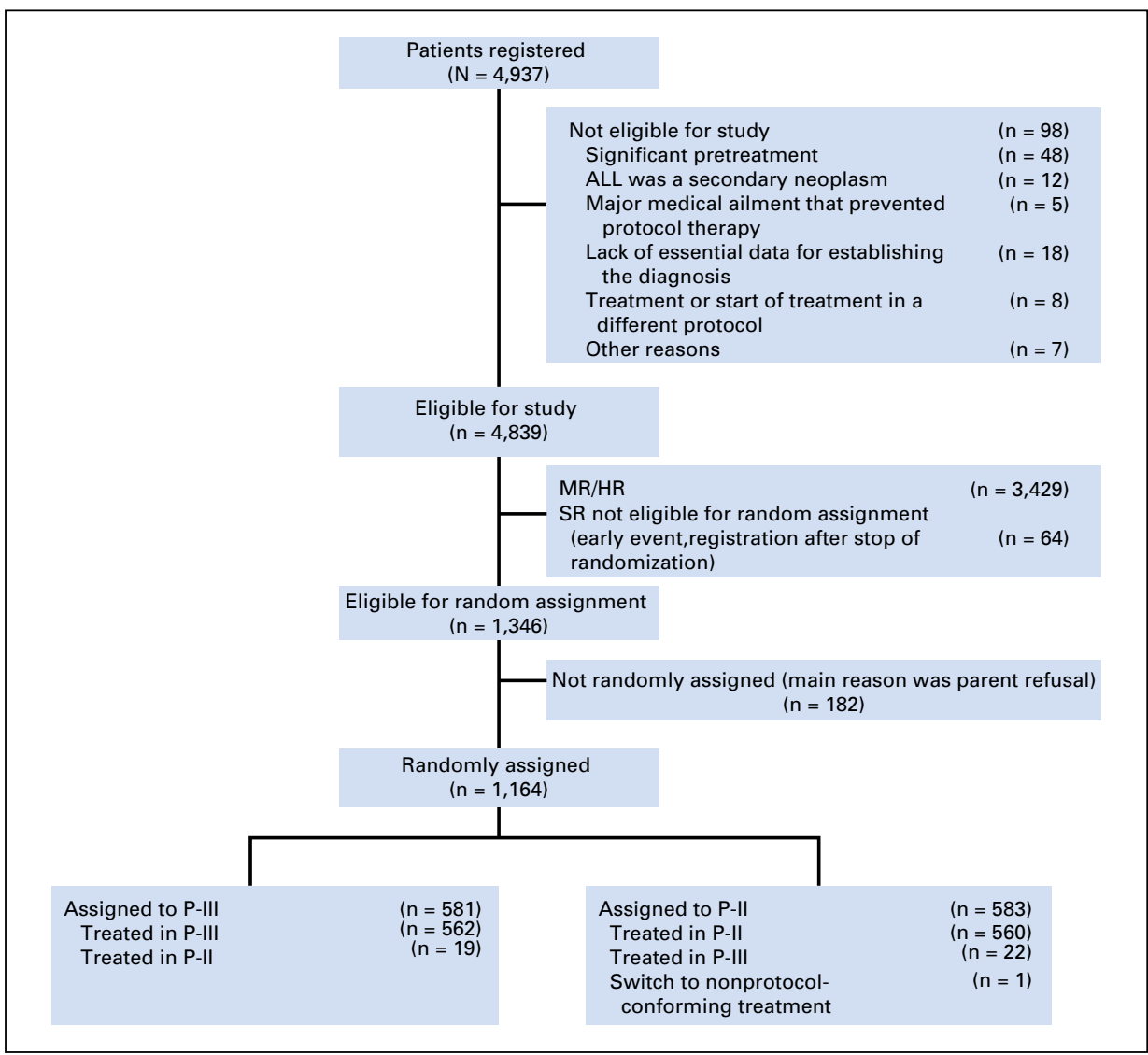

Fig 2. CONSORT diagram. Patient characteristics of randomly assigned and eligible nonrandomly assigned patients are listed in the Data Supplement. ALL, acute lymphoblastic leukemia; HR, high risk; MR, medium risk; P-II, protocol II; P-III, protocol III; SR, standard risk. 
AIEOP-BFM ALL 2000 trial after written informed consent from their guardians. Routine initial diagnostics were cytology, immunophenotyping, and molecular genetic screening for the presence of ETV6-RUNX1, BCR$A B L 1$, and KMT2A-AFF1 (MLL-AF4) fusion transcripts. Response assessment was performed by early cytologic assessment as well as by PCR-MRD on the basis of immunoglobulin and T-cell receptor gene rearrangements.. The respective standard procedures have been published previously. ${ }^{2,3,7,18-21}$

Good response or poor response to a 7-day prednisone prephase plus one intrathecal dose of methotrexate were defined as $<1.0 \times 10^{9} / \mathrm{L}$ and $\geq 1.0 \times 10^{9} / \mathrm{L}$ blasts in peripheral blood, respectively. Complete remission (CR) was defined as $<5 \%$ blasts in regenerating bone marrow at the end of induction treatment and the absence of extramedullary disease. Relapse was defined as either recurrence of $\geq 25 \%$ lymphoblasts in bone marrow or localized leukemic infiltrate at any site after having achieved CR.

Risk group assignment to the high-risk group in AIEOP-BFM ALL 2000 was based on genetic characterization of ALL (presence of $B C R-A B L 1$, $K M T 2 A-A F F 1)$ and slow cytologic and molecular response to treatment (prednisone poor response, no $\mathrm{CR}$ on day 33 , or $\mathrm{MRD} \geq 5 \times 10^{-4}$ on day 78). In the absence of the aforementioned high-risk criteria, patients were assigned to the SR group if MRD was negative on days 33 and 78 with at least two markers with a sensitivity of $1 \times 10^{-4}$. The remainder of the patients without high-risk criteria was assigned to the medium-risk group.

The study protocol was approved by the competent ethics committees of the national coordinating centers (San Gerardo Hospital, Monza, Italy; Hannover Medical School, Hannover, Germany; St Anna Children's Hospital, Vienna, Austria; and University Children's Hospital, Zürich, Switzerland) and registered as clinical trials (ClinicalTrials.gov identifiers: NCT00430118 [BFM] and NCT00613457 [AIEOP]). A data safety and monitoring committee periodically supervised the study progress.

\section{Randomization and Treatment}

Only SR patients were eligible for random assignment. They were assigned to receive either the experimental, less-intensive P-III or the standard P-II as delayed intensification. Random assignment was performed

\section{A}

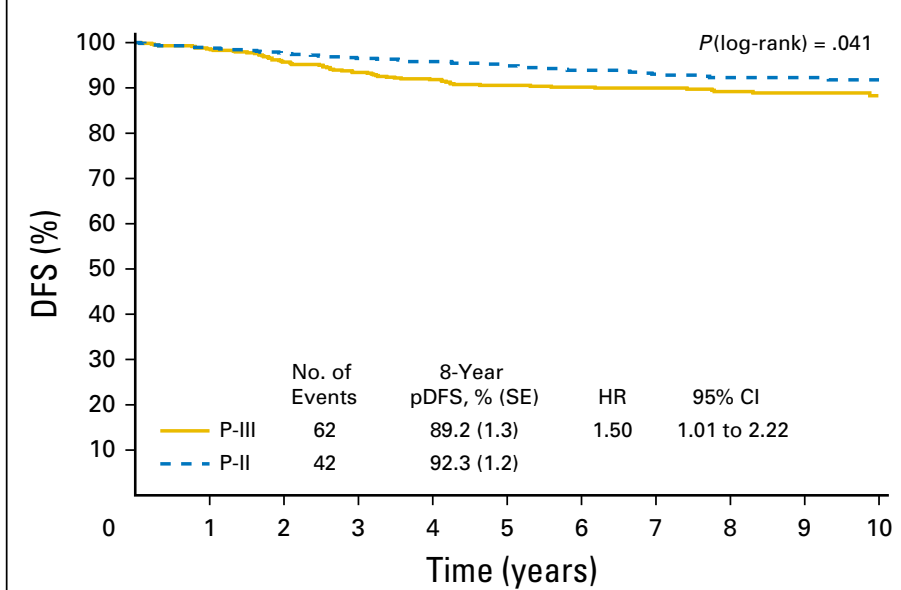

No. at risk:

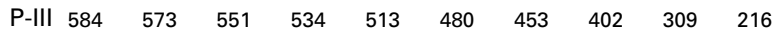

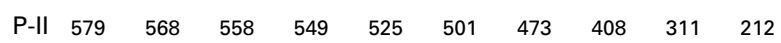

C

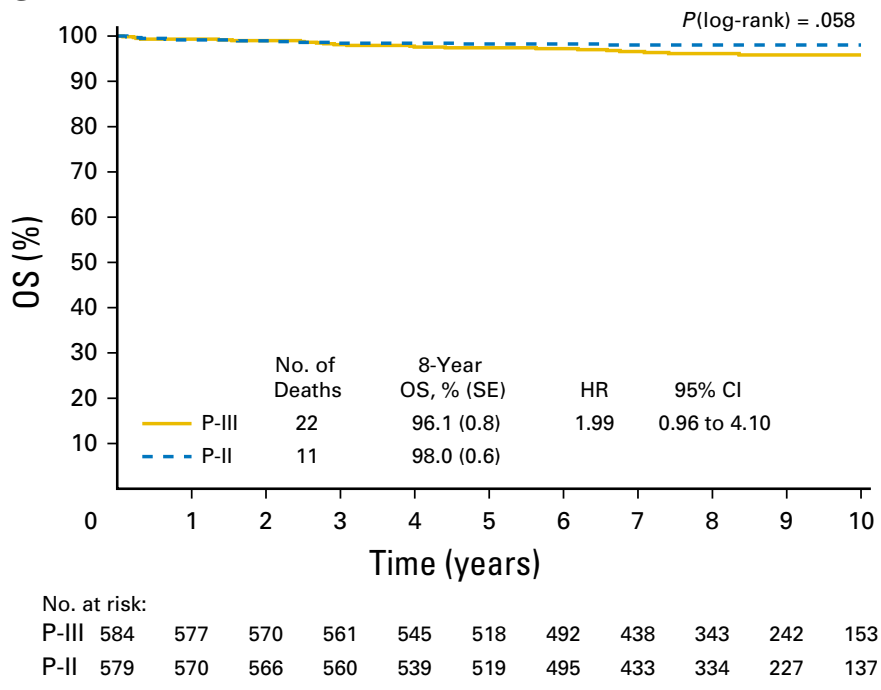

B

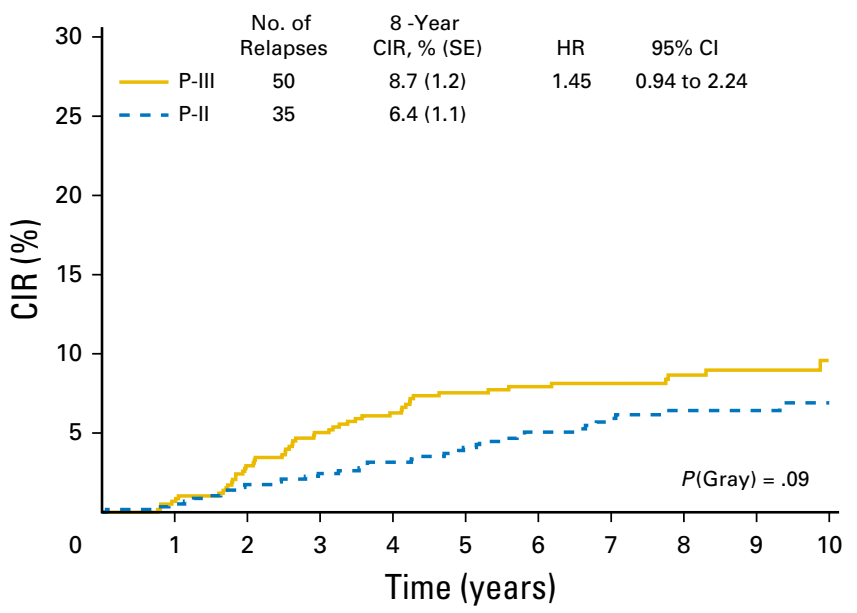

Fig 3. (A) Disease-free survival (DFS), (B) cumulative incidence of relapse (CIR), and (C) overall survival (OS) in the as-treated analysis. HR, hazard ratio; pDFS, probability of DFS; P-II, protocol II; P-III, protocol III. 


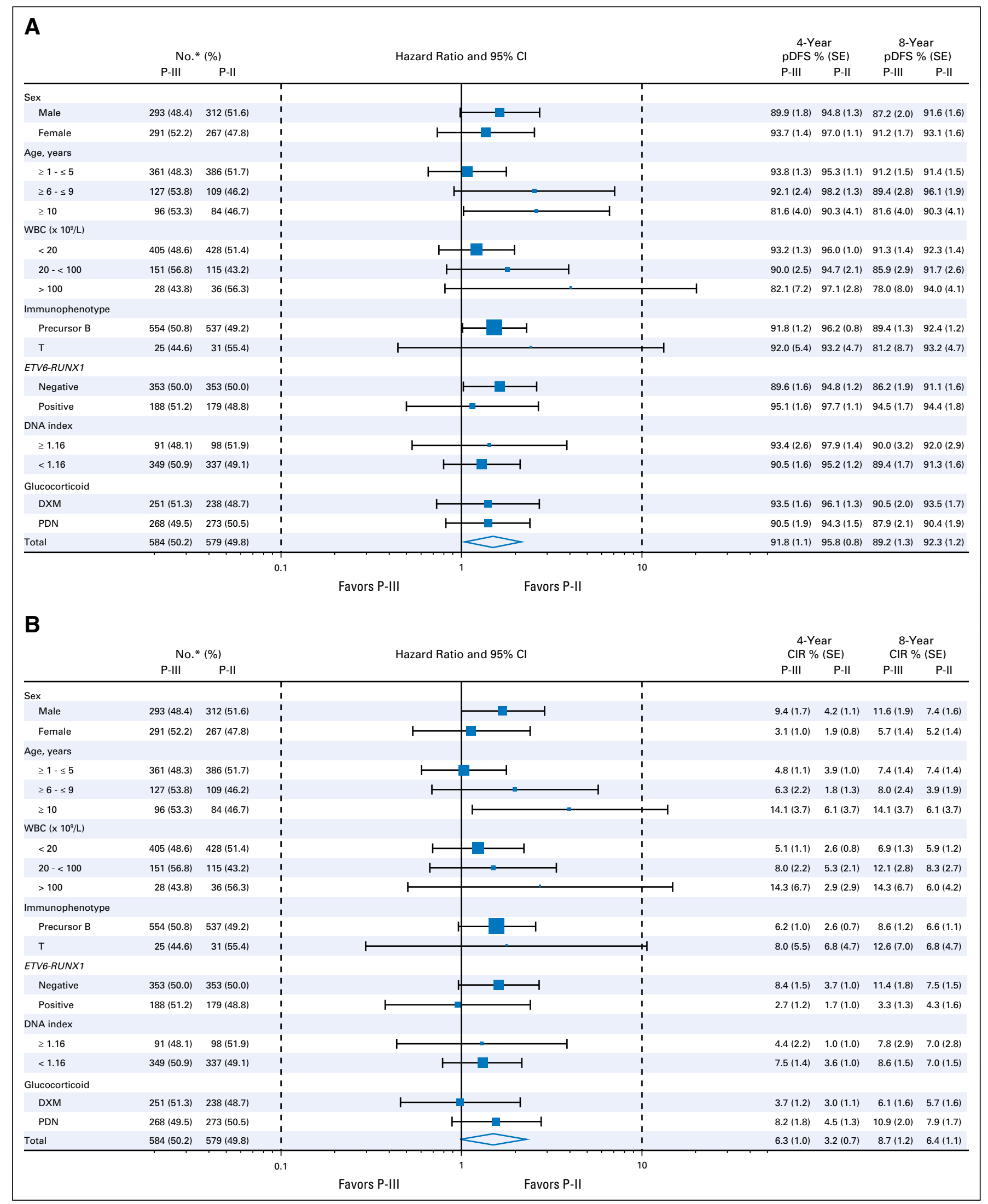

Fig 4. Forest plot of hazard ratio by patient subgroup for (A) disease-free survival (DFS), (B) cumulative incidence of relapse $(C I R)$, and $(C)$ overall survival $(O S)$. $\left(^{*}\right)$ Data refer to patients with successful investigation of the respective criteria. DXM, dexamethasone in induction treatment; pDFS, probability of DFS; PDN, prednisone in induction treatment; P-II, protocol II; P-III, protocol III; pOS, probability of OS. 


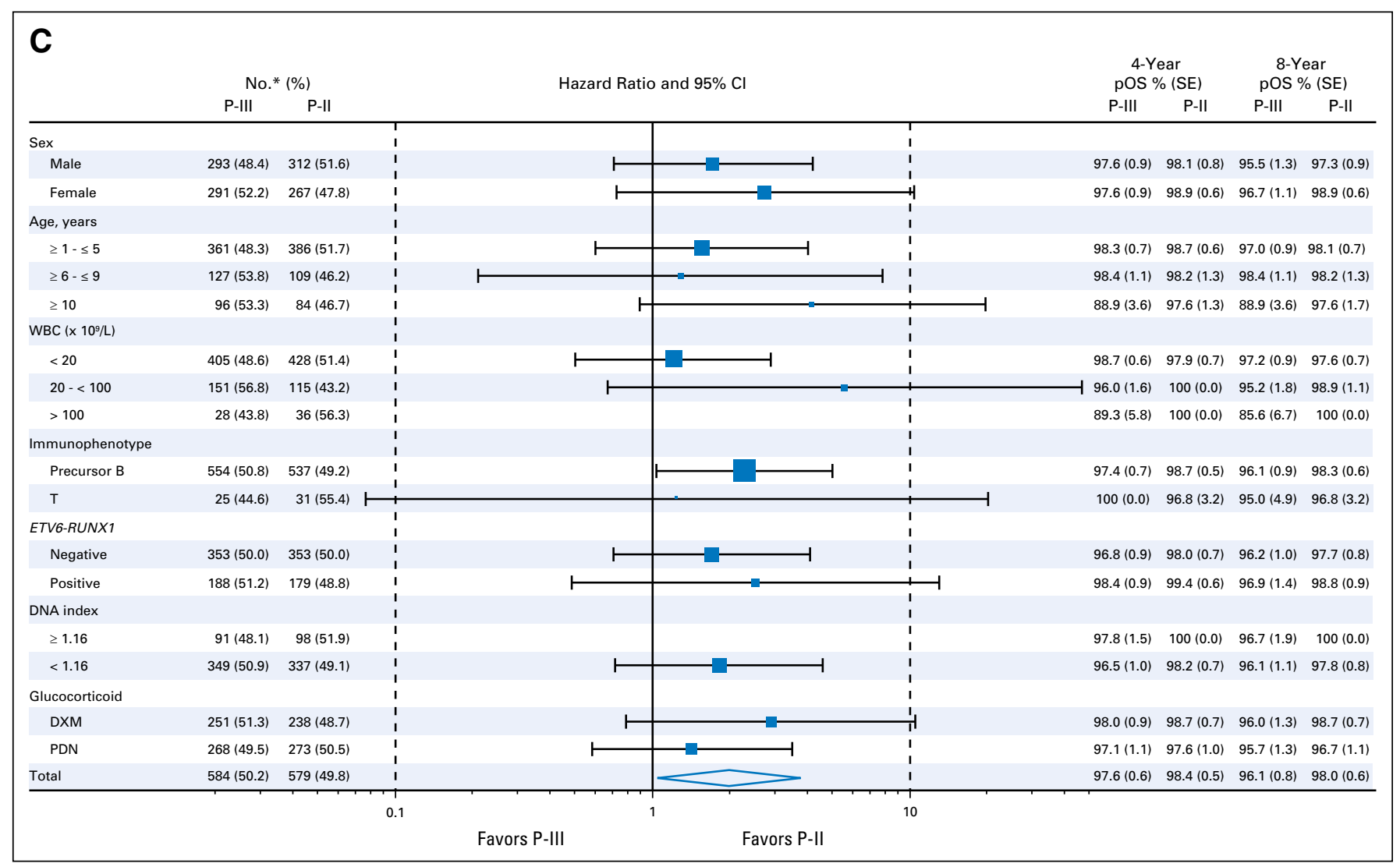

Fig 4. (Continued).

centrally by each country's data center in accordance with random permuted blocks. This randomization was stratified by allocation to a preceding random assignment (dexamethasone $v$ prednisone) and treatment center. ${ }^{22}$

P-III was shorter than P-II (duration, $28 v 49$ days), and its cumulative drug doses were reduced by $30 \%$ for dexamethasone and $50 \%$ for vincristine, doxorubicin, and cyclophosphamide compared with P-II. An outline of the SR treatment of AIEOP-BFM ALL 2000 is shown in Figure 1, with drug doses also listed in the Data Supplement. P-II and P-III are split into two parts. The first part lasts from day 1 to 29 in P-II and from day 1 to 14 in P-III, whereas the second part lasts from days 36 to 49 in P-II and from days 15 to 28 in P-III. Details on stratification, prognostic impact of MRD, and results of the random assignments during induction (dexamethasone $10 \mathrm{mg} / \mathrm{m}^{2} /$ day $v$ prednisone $60 \mathrm{mg} / \mathrm{m}^{2} /$ day) have been published previously. ${ }^{2,3,22}$ Criteria for cranial irradiation are listed in the Data Supplement.

\section{Statistical Analysis}

The primary outcome was disease-free survival (DFS) because all patients were in CR at random assignment. DFS was defined as the time from random assignment to the date of last follow-up or first event. Events were relapse, secondary neoplasm, or death from any cause. Secondary end points were overall survival (OS) defined as time to death from any cause, or last follow-up, and treatment-associated toxicities.

The objective of this randomized trial was to prove noninferiority of the reduced-intensity treatment compared with standard treatment. The main analysis was planned as a per-protocol evaluation of 4-year DFS. All randomly assigned patients were included in this analysis, but patients who switched trial arms were included in the treatment arm actually given. In addition, an intent-to-treat analysis was performed. The Kaplan-Meier method was used to estimate survival probabilities; differences between groups were compared by log-rank test. ${ }^{23}$ Cumulative incidence functions for competing events were constructed by the method of Kalbfleisch and Prentice and were compared with Gray's test. ${ }^{24,25}$ The Cox proportional hazard regression model was used for univariable and multivariable analyses. ${ }^{26}$ A sample size of 1,024 randomly assigned patients was considered appropriate to assess noninferiority $(\Delta<4 \%)$ with $90 \%$ power under the assumption of a $96 \% 4$-year DFS in the reference arm. Two interim analyses were planned 3 and 4 years after start of inclusion. For safety reasons, the interim analysis was a log-rank test of difference instead of the equivalence test planned for the final analysis.

\section{RESULTS}

\section{Patient Characteristics}

From July 1, 2000, to June 30 (July 31 for AIEOP), 2006, 4,937 patients were enrolled in AIEOP-BFM ALL 2000, of whom 98 were not eligible for evaluation (Fig 2). Among 1,346 SR patients eligible for random assignment, 182 were not assigned. The remaining 1,164 patients entered the randomized comparison; 581 children were allocated to the experimental arm P-III and 583 to the standard arm P-II. We observed no significant differences in initial patient characteristics among randomly assigned versus nonrandomly assigned patients as well as between the two arms analyzed by intention to treat or treatment given (Data Supplemental).

\section{Treatment Outcome}

After a median follow-up of 8.6 years, analysis per treatment given revealed a 4 -year probability of DFS ( $\mathrm{pDFS} \pm \mathrm{SE}$ ) rate of 
$91.8 \pm 1.1 \%$ in the reduced-intensity P-III arm $(\mathrm{n}=584)$ and of $95.8 \pm 0.8 \%$ in the P-II arm $(\mathrm{n}=579 ; P=.04)$ on the basis of the occurrence of 62 versus 42 events, respectively (Fig 3). The lower limit of the one-sided $95 \%$ CI for the pDFS rates was $-6.4 \%$, which is far below the noninferiority margin of $-4 \%,(P=.005$ for difference of the 4 -year pDFS estimates). The respective results at 8 years were $89.2 \pm 1.3 \%$ and $92.3 \pm 1.2 \%$ for $\mathrm{pDFS}(P=.041)$ and $8.7 \pm 1.2 \%$ and $6.4 \pm 1.1 \%$ for cumulative incidence of relapse (CIR; P-III $v$ P-II, $P=.09$; Fig 3). The 8-year OS rate was $96.1 \pm$ $0.8 \%$ and $98.0 \pm 0.6 \%(P=.06)$. The intent-to-treat analysis gave almost identical results (Data Supplement).

These differences of treatment outcome held for virtually all clinical and biologic subgroups, although in many subgroups the differences did not reach statistical significance (Figs $4 \mathrm{~A}$ to $4 \mathrm{C}$ ). ETV6-RUNX1 status and age at diagnosis represented the only exception. Eight-year pDFS rates of patients with ETV6-RUNX1- negative ALL were $86.2 \pm 1.9 \%$ versus $91.1 \pm 1.6 \%(P=.037)$ and for patients with ETV6-RUNX1-positive ALL, $94.5 \pm 1.7 \%$ versus $94.4 \pm 1.8 \%(P=.74)$ for P-III versus P-II, respectively (Figs 5A and $5 \mathrm{~B})$. Analogous results held for age at diagnosis. For patients 1 to 10 years of age, the 8 -year pDFS rate was $90.7 \pm 1.4 \%$ versus $92.4 \pm$ $1.2 \%(P=.26)$, and for patients age $\geq 10$ years, $81.6 \pm 4.0 \%$ versus $90.3 \pm 4.1 \%(P=.04)$ for P-III versus $\mathrm{P}$-II, respectively (Figs 5C and 5D).

The patterns of relapse with respect to time after diagnosis were different after P-III and P-II. The proportion of early relapses (ie, within 2.5 years after diagnosis) was higher in P-III than in P-II (P-III, 19 [38.0\%] of 50 relapses; P-II, 10 [28.6\%] of 35 relapses); the same occurred for late relapses $(2.5$ to $<5$ years after diagnosis; P-III, 23 [46.0\%] of 50 relapses; P-II, 10 [28.6\%] of 35 relapses), but a significantly higher proportion for very-late relapse ( $\geq 5$ years after diagnosis) was observed in patients treated

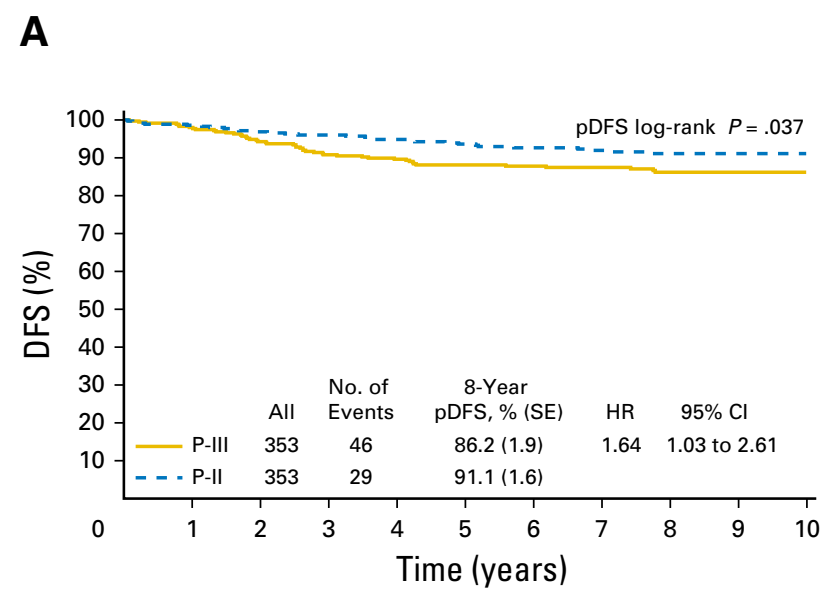

B

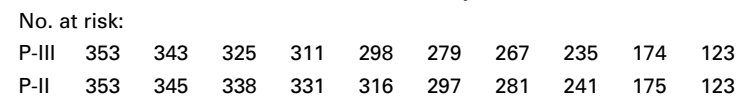

C

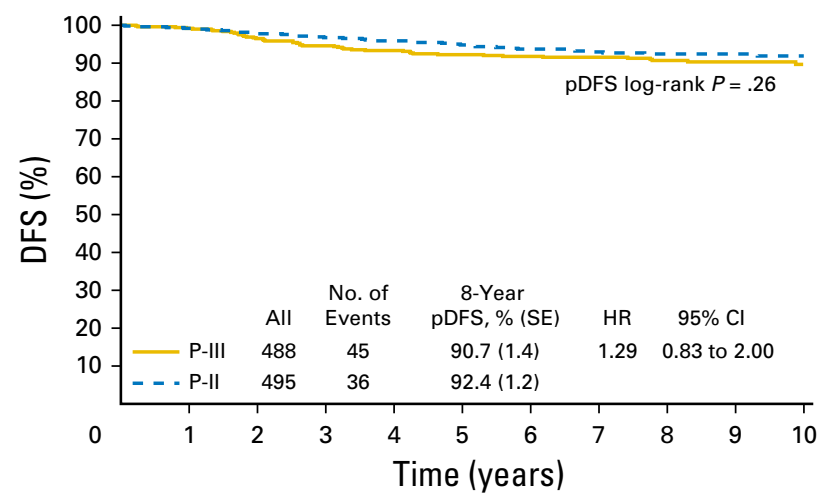

No. at risk:

$\begin{array}{lllllllllll}\text { P-III } & 488 & 481 & 464 & 450 & 437 & 412 & 396 & 355 & 275 & 199\end{array}$

$\begin{array}{lllllllllll}\text { P-II } & 495 & 488 & 480 & 471 & 452 & 433 & 414 & 358 & 279 & 196\end{array}$

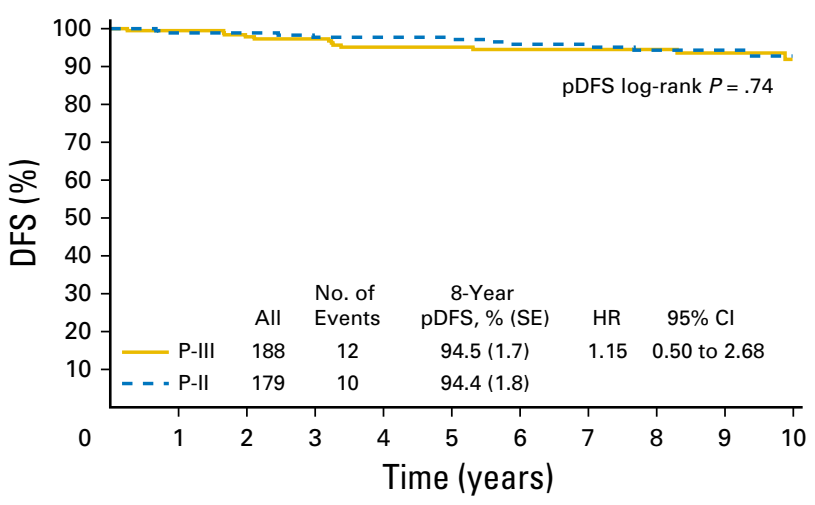

\begin{tabular}{lllllllllll}
\multicolumn{2}{l}{ No. at risk: } & & & & & & & & \\
P-III & 188 & 187 & 182 & 178 & 171 & 159 & 147 & 129 & 103 & 78 \\
P-II & 179 & 176 & 175 & 172 & 164 & 158 & 149 & 126 & 103 & 68
\end{tabular}

D

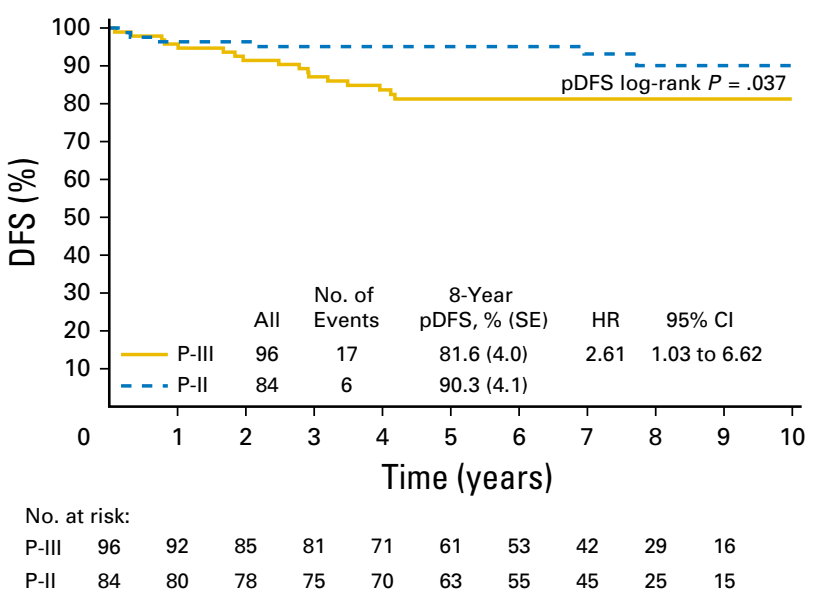

Fig 5. Disease-free survival (DFS) and cumulative incidence of relapse (CIR) according to ETV6-RUNX1 status and age at diagnosis (as treated). Data are shown for

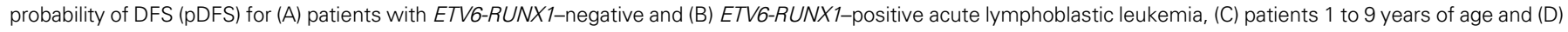

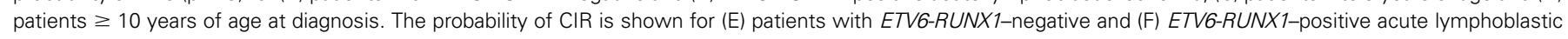
leukemia, (G) patients 1 to 9 years of age and $(\mathrm{H})$ patients $\geq 10$ years of age. HR, hazard ratio; OS, overall survival; P-II, protocol II; P-III, protocol III. 


\section{E}

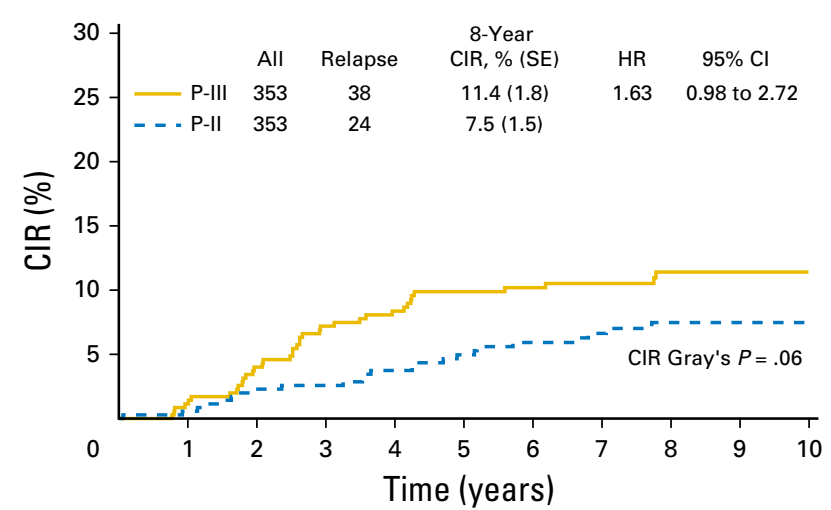

$\begin{array}{lllllllllll}\text { P-III } & 353 & 343 & 325 & 311 & 298 & 279 & 267 & 235 & 174 & 123 \\ \text { P-II } & 353 & 345 & 338 & 331 & 316 & 297 & 281 & 241 & 175 & 123\end{array}$

\section{G}

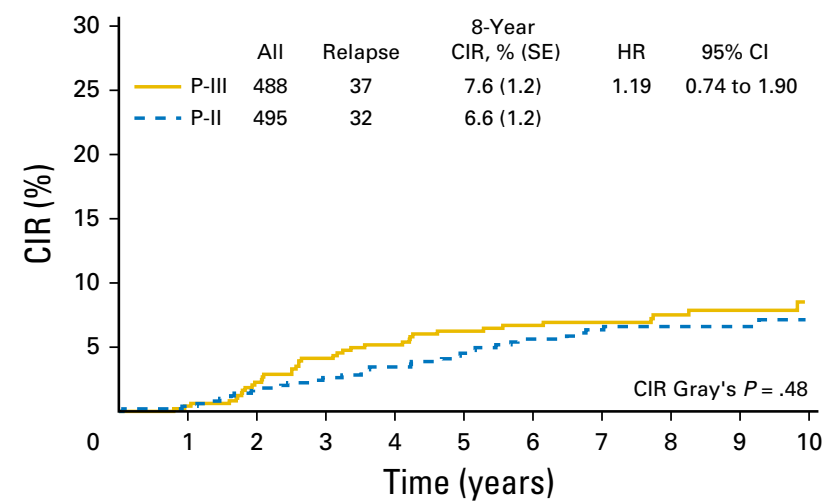

No. at risk:

$\begin{array}{lllllllllll}\text { P-III } & 488 & 481 & 464 & 450 & 437 & 412 & 396 & 355 & 275 & 199\end{array}$
$\mathbf{F}$

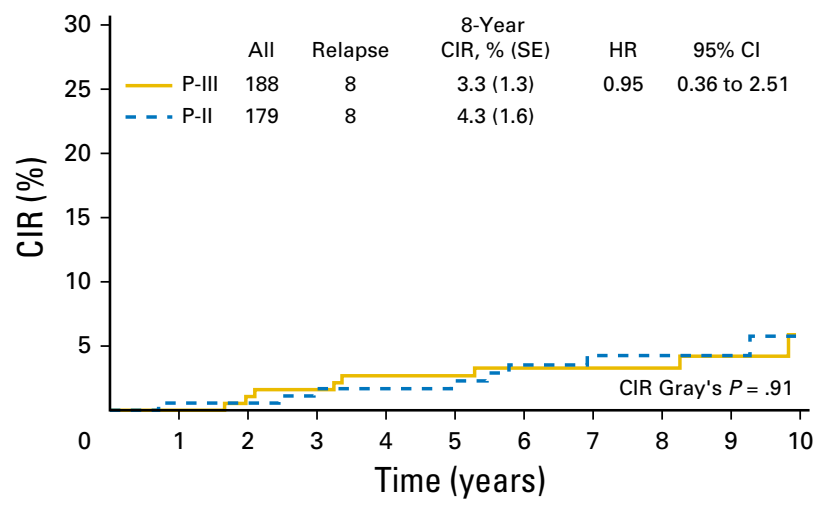

$\begin{array}{lllllllllll}\text { P-III } & 188 & 187 & 182 & 178 & 171 & 159 & 147 & 129 & 103 & 78 \\ \text { P-II } & 179 & 176 & 175 & 172 & 164 & 158 & 149 & 126 & 103 & 68\end{array}$

H

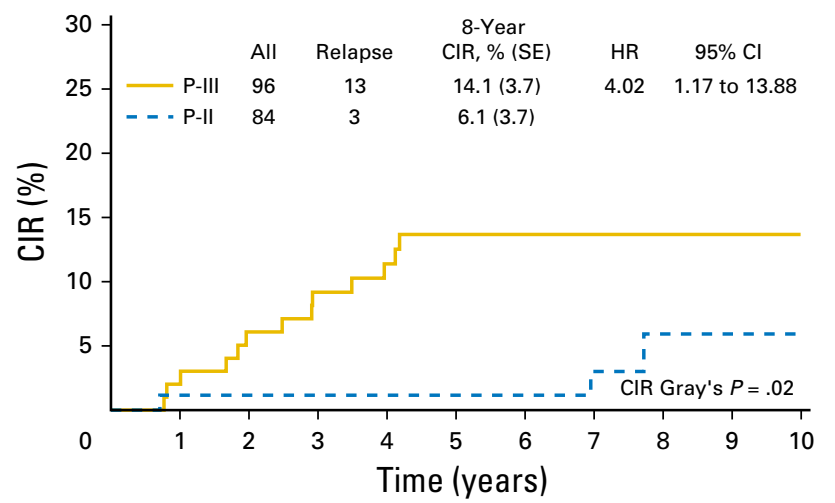

No. at risk:

$\begin{array}{lllllllllll}\text { P-III } & 96 & 92 & 85 & 81 & 71 & 61 & 53 & 42 & 29 & 16\end{array}$

Fig 5. (Continued).

with P-II (P-III, eight [16.0\%] of 50 relapses; P-II, 15 [42.9\%] of 35 relapses; $\left.P\left[\chi^{2}\right]=.022\right)$.

Secondary malignancies occurred in seven patients who received P-III (myelodysplastic syndrome $(n=2)$; CNS tumor $(\mathrm{n}=2)$; acute myeloid leukemia $(\mathrm{n}=2)$; and non-Hodgkin lymphoma $(\mathrm{n}=1)$ and in four patients who received P-II (myelodysplastic syndrome $(\mathrm{n}=2)$; acute myeloid leukemia $(\mathrm{n}=1)$; and solid tumor $(\mathrm{n}=1)$. This finding corresponds to an 8 -year cumulative incidence rate of secondary malignancy of $1.3 \pm 0.5 \%$ and $0.6 \pm 0.4 \%$ for patients given P-III and P-II, respectively $(P=.37$; Table 1).

An interaction between the initial corticosteroid in induction and outcome after random assignment has not been observed (Fig 4). Outcome by National Cancer Institute (NCI) criteria was an 8 -year pDFS rate of $91.6 \pm 1.4 \%$ versus $92.8 \pm 1.3 \%(P=.36)$, a CIR rate of $7.0 \pm 1.3 \%$ versus $6.2 \pm 1.2 \%(P=.49)$, and an 8 -year OS rate of $97.9 \pm 0.7 \%$ versus $98.1 \pm 0.7 \%(P=.77)$ for NCI SR for P-III and P-II, respectively. For NCI high risk, the
8 -year pDFS rate was $82.9 \pm 3.1 \%$ versus $90.4 \pm 2.7 \%(P=.041)$; CIR rate, $13.0 \pm 2.8 \%$ versus $7.5 \pm 2.5 \%(P=.07)$; and 8 -year OS rate, $91.3 \pm 2.3 \%$ versus $97.7 \pm 1.3 \%(P=.021)$ for P-III and P-II, respectively.

\section{Toxicity}

The incidence of death during remission was comparable, with $0.9 \pm 0.4 \%(\mathrm{n}=5)$ and $0.7 \pm 0.3 \%(\mathrm{n}=4)$ for P-III and P-II, respectively. The same applies for the number of adverse events, which was essentially the same in the two arms (Table 2). Life-threatening events, however, were slightly more likely to happen with P-II $(\mathrm{n}=10)$ than with P-III $(\mathrm{n}=7)$.

If analyzed separately for the first and the second phases of P-II and P-III, life-threatening adverse events were mostly observed in the first phase of P-II, whereas patients treated with P-III suffered from adverse events more frequently in the second phase of P-III. The median time needed until start of the second part of P-III or P-II was 15 days $(\mathrm{n}=184$ with data available) and 44 days 


\begin{tabular}{|c|c|c|c|c|c|c|c|c|}
\hline \multirow[b]{3}{*}{ Event } & \multicolumn{6}{|c|}{ Patient Group as Treated } & \multirow[b]{3}{*}{$P^{*}$} & \multirow[b]{3}{*}{$\mathrm{HR}(95 \% \mathrm{Cl})$} \\
\hline & \multicolumn{3}{|c|}{$P-I I I(n=584)$} & \multicolumn{3}{|c|}{$P-I I(n=579)$} & & \\
\hline & No. (\%) & $\begin{array}{l}\text { 4-Year Cumulative } \\
\text { Incidence, \% (SE) }\end{array}$ & $\begin{array}{l}\text { 8-Year Cumulative } \\
\text { Incidence, \% (SE) }\end{array}$ & No. (\%) & $\begin{array}{l}\text { 4-Year Cumulative } \\
\text { Incidence, \% (SE) }\end{array}$ & $\begin{array}{l}\text { 8-Year Cumulative } \\
\text { Incidence, \% (SE) }\end{array}$ & & \\
\hline Death in first CR & $5(0.9)$ & $0.9(0.4)$ & $0.9(0.4)$ & $4(0.7)$ & $0.7(0.3)$ & $0.7(0.3)$ & .75 & $1.24(0.33$ to 4.63$)$ \\
\hline All relapses & $50(8.6)$ & $6.3(1.0)$ & $8.7(1.2)$ & $35(6.0)$ & $3.2(0.7)$ & $6.4(1.1)$ & .09 & $1.45(0.94$ to 2.24$)$ \\
\hline Isolated BM & $26(4.5)$ & $2.8(0.7)$ & $4.8(0.9)$ & $20(3.5)$ & $1.8(0.6)$ & $3.8(0.8)$ & .34 & $1.33(0.74$ to 2.38$)$ \\
\hline Isolated CNS & $7(1.2)$ & $0.9(0.4)$ & $1.0(0.4)$ & $4(0.7)$ & $0.7(0.3)$ & $0.7(0.3)$ & .37 & 1.75 (0.51 to 5.97$)$ \\
\hline Isolated testes & $5(0.9)$ & $0.9(0.4)$ & $0.9(0.4)$ & $2(0.3)$ & $0.4(0.2)$ & $0.4(0.2)$ & .27 & $2.54(0.49$ to 13.13$)$ \\
\hline Combined BM/CNS & $6(1.0)$ & $0.7(0.3)$ & $0.9(0.4)$ & $4(0.7)$ & $0.4(0.2)$ & $0.6(0.3)$ & .52 & $1.52(0.43$ to 5.39$)$ \\
\hline Combined BM/othert & $6(1.0)$ & $1.0(0.4)$ & $1.0(0.4)$ & $3(0.5)$ & $0.0(0.0)$ & $0.6(0.3)$ & .31 & 2.05 (0.51 to 8.21$)$ \\
\hline Other relapses $\ddagger$ & $0(0.0)$ & $0.0(0.0)$ & $0.0(0.0)$ & $2(0.3)$ & $0.0(0.0)$ & $0.4(0.3)$ & - & - \\
\hline Secondary neoplasms & $7(1.2)$ & $1.0(0.4)$ & $1.3(0.5)$ & $4(0.7)$ & $0.4(0.2)$ & $0.6(0.4)$ & .37 & 1.76 (0.52 to 6.04$)$ \\
\hline
\end{tabular}

Abbreviations: BM, bone marrow; CR, complete response; HR, hazard ratio; P-II, protocol II; P-III, protocol III.

*Fisher's exact test was used for deaths and Gray's test for relapses and secondary neoplasms.

tOther relapse sites comprise testis, eye, lymph node, and bone.

¥Other relapses comprise abdominal and ovary.

( $\mathrm{n}=173$ with data available), respectively; 15 and 36 days were the expected time according to protocol, respectively.

\section{DISCUSSION}

The attempt to reduce chemotherapy burden by choosing P-III as a lower-intensity delayed intensification in patients considered to be at low relapse risk did not succeed because outcome results were inferior to those obtained with the standard treatment. DFS data showed a significantly higher proportion of events in the experimental arm, especially for patients who were ETV6-RUNX1 negative or $\geq 10$ years of age. In addition, the expected benefit of reduced-intensity treatment in terms of acute toxicity and rates of second malignancy could not be demonstrated. In fact, toxicity seems to be virtually the same in both treatment arms. A possible explanation for this observation is a less stringent handling of patients in P-III by the treating physicians, which is probably attributable to an underestimation of the impact of this treatment element. This hypothesis is supported by the significantly shorter treatment delay between both parts of the respective treatment phase ( 2 days in P-III $v 14$ days in P-II). Patients treated with P-III thus experienced a higher dose density than patients treated with P-II.

Considering the higher number of relapses in patients treated with the less-intensive P-III, at least a subpopulation in the SR group was fairly undertreated with P-III. This refers particularly to the inferior outcome of patients $\geq 10$ years of age and those with ETV6-RUNX1 negative precursor B-cell ALL, which suggests a greater effect in subsets with known unfavorable characteristics despite the very favorable early response depicted by highly sensitive PCR-MRD.

In patients in the prognostically more favorable subgroups, such as those with ETV6-RUNX1-positive ALL and those 1 to 6 years of age at diagnosis, the hazard ratios for the incidence of relapse did not indicate an increase in the relapse rate, with the disadvantage of treatment reduction possibly being negligible (Fig $4 \mathrm{~B})$. The same can be seen in patients treated with dexamethasone during induction.

\begin{tabular}{|c|c|c|c|c|c|c|}
\hline \multirow[b]{3}{*}{ Adverse Events } & \multicolumn{6}{|c|}{ Adverse Event, No. (\%)* } \\
\hline & \multicolumn{2}{|c|}{ Non-Life Threatening } & \multicolumn{2}{|c|}{ Life Threatening $†$} & \multicolumn{2}{|c|}{ Fatal } \\
\hline & P-III & $P-\| \ddagger$ & P-III & P-IIf & P-III & P-II‡ \\
\hline All & $25(4.2)$ & $29(5.0)$ & $7(1.2)$ & $10(1.7)$ & $4(0.7)$ & $2(0.4)$ \\
\hline Infection related & $8(1.3)$ & $9(1.6)$ & $4(0.7)$ & $5(0.9)$ & $3(0.5)$ & - \\
\hline Not infection related & $17(2.9)$ & $20(3.5)$ & $3(0.5)$ & $5(0.9)$ & $1(0.2)$ & $2(0.4)$ \\
\hline \multicolumn{7}{|c|}{$\begin{array}{l}\text { NOTE. All events are included that were related to therapy and occurred during or after delayed intensification before the start of maintenance treatment. Events were } \\
\text { captured according to a list of clinically relevant events specified in the trial protocol. Infectious complications cover life-threatening infections, systemic/invasive fungal } \\
\text { infections, and severe local infections. Noninfectious events comprise pancreatitis, deep venous thrombosis, sinus venous thrombosis, cardiac insufficiency grade } 3 / 4 \\
\text { and cardiac arrhythmias grade } 3 / 4 \text {, seizures, alteration of consciousness grade } 3 / 4 \text {, paresis/paresthesia, cerebral hemorrhage, cerebral stroke, severe psychosis that } \\
\text { requires medication, generalized exfoliative dermatitis, GI hemorrhage grade } 3 / 4, G \text { ulcer grade } 3 / 4 \text {, intestinal perforation, severe hepatic failure, veno-occlusive disease, } \\
\text { acute renal failure that requires dialysis, significant chronic renal failure, drug-induced diabetes mellitus with hyperglycemic shock, allergic reaction with shock } \\
\text { symptoms, osteonecrosis, fracture, and other events assessed as clinically relevant by the investigators. } \\
\text { Abbreviations: P-II, protocol II; P-III, protocol III. } \\
{ }^{*} \text { Percentages are related to the total number of patients as treated in the respective arm (P-III, } n=584 ; \text { P-II, n = 579). } \\
\text { †An adverse event was considered life threatening if its occurrence placed the patient at immediate risk of death. An adverse event that might have caused death, if it } \\
\text { had occurred in a more severe form, was not considered life threatening. } \\
\ddagger \text { Patient group as treated. }\end{array}$} \\
\hline
\end{tabular}


The current randomization was designed on the basis of the results of the ALL-BFM 81 and ALL-BFM 83 trials, which were the first by our study group that introduced a reintensification regimen called protocol 3 in the SR groups. Furthermore, these two trials documented the distinct importance of delayed intensification for the prevention of relapse. ${ }^{1,18,27}$ Hence, in subsequent trials, reintensification became an integral element of ALL treatment by using the more intensive version of reintensification (P-II) from ALL-BFM 86. ${ }^{1,28}$ In the current trial (AIEOP-BFM ALL 2000), P-III was randomized against the latter to find an approximation toward a less-intensive, but still-effective treatment.

Most recently, the Dutch Childhood Oncology Group (DCOG) reported the results of its study DCOG ALL10 with nonrandomized treatment reduction for SR patients during delayed intensification. ${ }^{11}$ The risk stratification criteria in this trial were similar to those in our trial. Treatment of SR patients was also comparable except for the reduced delayed intensification phase, which was considerably more reduced in intensity in that trial than in P-III. With reduced-intensity chemotherapy at a median follow-up of 80 months, 194 patients had a 5-year pEFS rate of $93.1 \pm 1.9 \%$ and 5-year CIR rate of $6.4 \pm 1.85 \%$. The outcome results in this rather small cohort were interpreted as improvement compared with historical controls of the DCOG, and therapy reduction was declared as safe by study criteria. However, the pEFS was inferior, although not significant, to the results of the historical MRD-SR group as reported by the International BFM Study Group $^{5}$ (5-year pEFS, 98\%; SE, 2\%; $\mathrm{n}=55 ; P=.08$ ).

Of note, the rates of 5 -year pEFS and 5-year CIR in the DCOG study were in between the respective results of P-III (5-year pDFS, $90.6 \pm 1.2 \%[\Delta=2.5 \%] ; 5$-year CIR, $7.5 \pm 1.1 \%[\Delta=1.1 \%])$ and P-II (5-year pDFS, $94.9 \pm 0.9 \%[\Delta=1.8 \%]$; 5-year CIR, $4.1 \pm$ $0.8 \%$ [ $\Delta=-2.3 \%])$ of the AIEOP-BFM ALL 2000 trial. This finding leaves open the question of whether the reduced intensity in the DCOG study is really noninferior to standard treatment with P-II.

In the study Malaysia-Singapore ALL 2003 s, risk stratification was likewise based on PCR-MRD by basically using the same risk stratification criteria as in our and the DCOG ALL10 protocols. SR patients were treated with a nonrandomized reduced therapy on the backbone of the ALL-Intercontinental BFM 2002 protocol. $^{13,29}$ The treatment included a three-drug induction without anthracyclines as well as a reduced reinduction roughly comparable to P-III as reported here. With a rather short median follow-up of 3.38 years, the SR group of 172 patients had a 6 -year pEFS rate of $93.2 \pm 4.1 \%$ and a 6-year OS rate of $95.4 \pm 3.3 \%$, which depicts major improvement with the reduced-intensity treatment approach compared with preceding results of this study group along with a lower incidence of fatal and/or life-threatening treatment-related events. With the lack of a randomized approach, however, these improvements hardly could be differentiated from general improvements in quality of treatment and supportive care.

A randomized approach was chosen in the protocol of the British study group trial UKALL 2003, where 521 low-risk patients assessed by NCI criteria and PCR-MRD were randomly assigned to receive either one or two delayed intensification courses. ${ }^{12}$ With a median follow-up of 57 months, a difference in 5-year pEFS rate of $1.1 \%$ was reported $(94.4 \% v 95.5 \%)$. The $95 \%$ CI of the difference was $-5.6 \%$ to $2.5 \%$. The authors concluded that the primary end point of the randomization (to rule out a 7\% reduction in EFS) was achieved. With the noninferiority margin of $4 \%$ in the current trial, this would not be true. Defining a reasonable noninferiority margin is always a matter of debate.

Another randomized treatment question about delayed intensification was asked by the US Children's Oncology Group. Comparability with the aforementioned trials and our study, however, is even more limited because MRD was not used for risk stratification. ${ }^{10}$ The authors demonstrated that the addition of a second delayed intensification in the treatment schedule of NCI SR patients with rapid early cytologic marrow response did not offer an advantage in terms of 5-year pEFS and OS rate (90.9 \pm $1.3 \%$ and $97.1 \pm 0.8 \% v 90.5 \pm 1.3 \%$ and $95.4 \pm 3.8 \%$ for single and double delayed intensification, respectively).

In summary, both of the latter-cited trials-UKALL 2003 and COG 1991 - revealed the feasibility of treatment reduction in patients with the most favorable prognosis in a randomized approach. Discrepancies with the results presented here might be explained by a fairly different treatment approach. A higher treatment intensity in the reduced intensity arm with single delayed intensification, which is comparable with P-II in the current study, presumably is a critical threshold that has not been reached. Differences in the risk stratification approaches also hamper comparability with the current trial. ${ }^{11,13,28}$

As a future perspective, a constant refining of biologic subtypes and treatment response is warranted to ensure the best possible treatment stratification throughout study groups. Insertion of novel drugs in chemotherapeutic regimens and implementation of targeted therapies, such as leukemia-specific antibodies, hopefully will take their place in the treatment of SR leukemia and thus enable a reduction of conventional drugs. In particular, by sparing anthracyclines and alkylating agents, the reduction of acute and long-term toxicity could be achieved. However, as the results of the presented trial demonstrate, future development should be implemented carefully by means of randomized trials designed to recruit sufficiently large cohorts to enable detection of clinically relevant differences.

\section{AUTHORS' DISCLOSURES OF POTENTIAL CONFLICTS} OF INTEREST

Disclosures provided by the authors are available with this article at jco.org.

\section{AUTHOR CONTRIBUTIONS}

Conception and design: Martin Schrappe, Martin Zimmermann, Andrea Biondi, Franco Locatelli, Carmelo Rizzari, Maria Grazia Valsecchi, Felix Niggli, Georg Mann, Rita Beier, Wolf-Dieter Ludwig, Giuseppe Basso,

Valentino Conter

Collection and assembly of data: Martin Schrappe, Andrea Biondi, Anja Möricke, Franco Locatelli, Gunnar Cario, Carmelo Rizzari, Andishe Attarbaschi, Maria Grazia Valsecchi, Claus R. Bartram, Elena Barisone, Felix Niggli, Charlotte Niemeyer, Anna Maria Testi, Georg Mann, Ottavio Ziino, Beat Schäfer, Renate Panzer-Grümayer, Rita Beier, Rosanna Parasole, Gudrun Göhring, Wolf-Dieter Ludwig, Fiorina Casale, Paul-Gerhardt Schlegel, Giuseppe Basso, Valentino Conter

Data analysis and interpretation: Martin Schrappe, Kirsten Bleckmann, Martin Zimmermann, Anja Möricke, Valentino Conter

Manuscript writing: All authors

Final approval of manuscript: All authors

Accountable for all aspects of the work: All authors 


\section{REFERENCES}

1. Möricke A, Zimmermann M, Reiter A, et al: Long-term results of five consecutive trials in childhood acute lymphoblastic leukemia performed by the ALL-BFM study group from 1981 to 2000. Leukemia 24:265-284, 2010

2. Conter $V$, Bartram $C R$, Valsecchi $M G$, et al: Molecular response to treatment redefines all prognostic factors in children and adolescents with B-cell precursor acute lymphoblastic leukemia: Results in 3184 patients of the AIEOP-BFM ALL 2000 study. Blood 115:3206-3214, 2010

3. Schrappe $M$, Valsecchi MG, Bartram CR, et al: Late $M R D$ response determines relapse risk overall and in subsets of childhood T-cell ALL: Results of the AIEOP-BFM-ALL 2000 study. Blood 118:2077-2084, 2011

4. Pui $C H$, Pei $D$, Sandlund JT, et al: Long-term results of St Jude Total Therapy Studies 11, 12, 13A, $13 \mathrm{~B}$, and 14 for childhood acute lymphoblastic leukemia. Leukemia 24:371-382, 2010

5. van Dongen JJ, Seriu T, Panzer-Grümayer ER, et al: Prognostic value of minimal residual disease in acute lymphoblastic leukaemia in childhood. Lancet 352:1731-1738, 1998

6. Flohr T, Schrauder A, Cazzaniga G, et al: Minimal residual disease-directed risk stratification using real-time quantitative PCR analysis of immunoglobulin and T-cell receptor gene rearrangements in the international multicenter trial AIEOP-BFM ALL 2000 for childhood acute lymphoblastic leukemia. Leukemia 22:771-782, 2008

7. van Dongen JJ, van der Velden VH, Brüggemann $M$, et al: Minimal residual disease diagnostics in acute lymphoblastic leukemia: Need for sensitive, fast, and standardized technologies. Blood 125:3996-4009, 2015

8. O'Connor D, Bate J, Wade R, et al: Infectionrelated mortality in children with acute lymphoblastic leukemia: An analysis of infectious deaths on UKALL2003. Blood 124:1056-1061, 2014

9. Schmiegelow K, Attarbaschi A, Barzilai S, et al: Consensus definitions of 14 severe acute toxic effects for childhood lymphoblastic leukaemia treatment: A Delphi consensus. Lancet Oncol 17: e231-e239, 2016

10. Matloub Y, Bostrom BC, Hunger SP, et al: Escalating intravenous methotrexate improves event-free survival in children with standard-risk acute lymphoblastic leukemia: A report from the Children's Oncology Group. Blood 118:243-251, 2011

11. Pieters $R$, de Groot-Kruseman $H$, Van der Velden $V$, et al: Successful therapy reduction and intensification for childhood acute lymphoblastic leukemia based on minimal residual disease monitoring: Study ALL10 from the Dutch Childhood Oncology Group. J Clin Oncol 34:2591-2601, 2016

12. Vora A, Goulden N, Wade R, et al: Treatment reduction for children and young adults with low-risk acute lymphoblastic leukaemia defined by minimal residual disease (UKALL 2003): A randomised controlled trial. Lancet Oncol 14:199-209, 2013

13. Yeoh $A E$, Ariffin $H$, Chai EL, et al: Minimal residual disease-guided treatment deintensification for children with acute lymphoblastic leukemia: Results from the Malaysia-Singapore acute lymphoblastic leukemia 2003 study. J Clin Oncol 30:2384-2392, 2012

14. Brisco MJ, Condon J, Hughes E, et al: Outcome prediction in childhood acute lymphoblastic leukaemia by molecular quantification of residual disease at the end of induction. Lancet 343:196-200, 1994

15. Henze G, Langermann HJ, Brämswig J, et al: The BFM 76/79 acute lymphoblastic leukemia therapy study (author's transl) [in German]. Klin Padiatr 193:145-154, 1981

16. Henze G, Langermann HJ, Fengler $R$, et al: Acute lymphoblastic leukemia therapy study BFM 79/81 in children and adolescents: Intensified reinduction therapy for patients with different risk for relapse [in German]. Klin Padiatr 194:195-203, 1982

17. Riehm H, Feickert HJ, Schrappe M, et al: Therapy results in five ALL-BFM studies since 1970: Implications of risk factors for prognosis. Haematol Blood Transfus 30:139-146, 1987

18. Riehm $H$, Reiter $A$, Schrappe $M$, et al: Corticosteroid-dependent reduction of leukocyte count in blood as a prognostic factor in acute lymphoblastic leukemia in childhood (therapy study ALLBFM 83) [in German]. Klin Padiatr 199:151-160, 1987
19. Bene MC, Castoldi G, Knapp W, et al: Proposals for the immunological classification of acute leukemias. Leukemia 9:1783-1786, 1995

20. van der Velden VH, Cazzaniga G, Schrauder A, et al: Analysis of minimal residual disease by $\mathrm{lg} / \mathrm{TCR}$ gene rearrangements: Guidelines for interpretation of real-time quantitative PCR data. Leukemia 21: 604-611, 2007

21. van der Does-Van den Berg $A$, Bartram $C R$, Basso G, et al: Minimal requirements for the diagnosis, classification, and evaluation of the treatment of childhood acute lymphoblastic leukemia (ALL) in the "BFM Family" Cooperative Group. Med Pediatr Oncol 20:497-505, 1992

22. Möricke A, Zimmermann M, Valsecchi MG, et al: Dexamethasone vs prednisone in induction treatment of pediatric ALL: Results of the randomized trial AIEOPBFM ALL 2000. Blood 127:2101-2112, 2016

23. Kaplan EL, Meier P: Nonparametric estimation from incomplete observations. J Am Stat Assoc 53: 457-485, 1958

24. Kalbfleisch JPR. The Statistical Analysis of Failure Time Data. New York, NY, Wiley, 1980

25. Gray RJ: A class of k-sample tests for comparing the cumulative incidence of a competing risk. Ann Stat 16:1141-1154, 1988

26. Cox D: Regression models and life tables. J R Stat Soc B 34:187-220, 1972

27. Schrappe M, Beck J, Brandeis WE, et al: Treatment of acute lymphoblastic leukemia in childhood and adolescence: Results of the multicenter therapy study ALL-BFM 81 [in German]. Klin Padiatr 199:133-150, 1987

28. Möricke $A$, Reiter $A$, Zimmermann $M$, et al: Risk-adjusted therapy of acute lymphoblastic leukemia can decrease treatment burden and improve survival: Treatment results of 2169 unselected pediatric and adolescent patients enrolled in the trial ALL-BFM 95. Blood 111:4477-4489, 2008 [Erratum: Blood 113:4478, 2009]

29. Stary J, Zimmermann M, Campbell M, et al: Intensive chemotherapy for childhood acute lymphoblastic leukemia: Results of the randomized intercontinental trial ALL IC-BFM 2002. J Clin Oncol 32: 174-184, 2014

\section{Affiliations}

Martin Schrappe, Kirsten Bleckmann, Anja Möricke, and Gunnar Cario, University Hospital Schleswig-Holstein, Campus Kiel, Kiel; Martin Zimmermann and Gudrun Göhring, Hannover Medical School, Hannover; Claus R. Bartram, Institute of Human Genetics, Ruprecht-Karls University, Heidelberg; Charlotte Niemeyer, University of Freiburg, Freiburg; Rita Beier, University Hospital, Essen; WolfDieter Ludwig, HELIOS Medical Center Berlin-Buch, Berlin; Paul-Gerhardt Schlegel, University of Würzburg, Würzburg, Germany; Andrea Biondi, Carmelo Rizzari, Maria Grazia Valsecchi, and Valentino Conter, University of Milano-Bicocca; Andrea Biondi, Carmelo Rizzari, and Valentino Conter, Ospedale San Gerardo, Monza; Franco Locatelli, University of Pavia and Istituto di Ricovero e Cura a Carattere Scientifico Ospedale Pediatrico Bambino Gesù; Anna Maria Testi, University La Sapienza, Rome; Elena Barisone, Regina Margherita Children's Hospital, Turin; Ottavio Ziino, Azienda di Rilievo Nazionale ad Alta Specializzazione Ospedali Civico Di Cristina, Palermo; Rosanna Parasole, Santobono-Pausilipon Children's Hospital; Fiorina Casale, University of Naples, Naples; Giuseppe Basso, University of Padova, Padova, Italy; Andishe Attarbaschi, Georg Mann, and Renate Panzer-Grümayer, St Anna Children's Hospital and Medical University of Vienna, Vienna, Austria; and Felix Niggli and Beat Schäfer, University Hospital Zürich, Zürich, Switzerland.

\section{Support}

Associazione Italiana di Ematologia e Oncologia Pediatrica (AIEOP) was supported by Comitato Maria Letizia Verga and Fondazione Tettamanti; Fondazione Città della Speranza; Fondazione Cariparo; Associazione Gian Franco Lupo; Associazione Italiana per la Ricerca sul Cancro (A.B., M.G.V.); Special grant 5 x 1.000 (F.L.); Fondazione Cariplo (A.B.); and Ministero dell'Istruzione, Università e Ricerca (A.B., F.L., AIRC [Assocciazone Italiana Ricera sul Cancro] IG [investigator grant] 17200). Berlin-Frankfurt-Münster (BFM) was supported by Grants No. 50-2698 Schr1 and 50-2410 Ba7 from Deutsche Krebshilfe eV; Grant No. OCS 1230-02-2002 from Oncosuisse/Krebsforschung Schweiz; and St Anna Kinderkrebsforschung. 


\section{AUTHORS' DISCLOSURES OF POTENTIAL CONFLICTS OF INTEREST}

Reduced-Intensity Delayed Intensification in Standard-Risk Pediatric Acute Lymphoblastic Leukemia Defined by Undetectable Minimal Residual Disease: Results of an International Randomized Trial (AIEOP-BFM ALL 2000)

The following represents disclosure information provided by authors of this manuscript. All relationships are considered compensated. Relationships are self-held unless noted. I = Immediate Family Member, Inst = My Institution. Relationships may not relate to the subject matter of this manuscript. For more information about ASCO's conflict of interest policy, please refer to www.asco.org/rwc or ascopubs.org/jco/site/ifc.

\section{Martin Schrappe}

Honoraria: PrIME Oncology

Research Funding: Sigma Tau Pharmaceuticals (Inst), Baxter (Inst), Medac (Inst)

Kirsten Bleckmann

Travel, Accommodations, Expenses: Jazz Pharmaceuticals

Martin Zimmermann

No relationship to disclose

Andrea Biondi

No relationship to disclose

Anja Möricke

Honoraria: Baxalta, Jazz Pharmaceuticals

Travel, Accommodations, Expenses: Jazz Pharmaceuticals

Franco Locatelli

Honoraria: Amgen, Miltenyi Biotec, Mallinckrodt

Consulting or Advisory Role: Amgen

Research Funding: Neovii Biotech

Travel, Accommodations, Expenses: Medac

\section{Gunnar Cario}

No relationship to disclose

\section{Carmelo Rizzari}

Honoraria: Jazz Pharmaceuticals

Consulting or Advisory Role: TEVA Pharmaceuticals Industries, Jazz Pharmaceuticals, Amgen

Andishe Attarbaschi

Consulting or Advisory Role: Jazz Pharmaceuticals

Travel, Accommodations, Expenses: Jazz Pharmaceuticals, Amgen, Pfizer

Maria Grazia Valsecchi

No relationship to disclose

Claus R. Bartram

No relationship to disclose

Elena Barisone

Travel, Accommodations, Expenses: Jazz Pharmaceuticals

Felix Niggli

No relationship to disclose

\section{Charlotte Niemeyer}

Consulting or Advisory Role: Celgene, Novartis

Anna Maria Testi

No relationship to disclose

Georg Mann

No relationship to disclose

Ottavio Ziino

Travel, Accommodations, Expenses: Jazz Pharmaceuticals

Beat Schäfer

No relationship to disclose

Renate Panzer-Grümayer

No relationship to disclose

Rita Beier

Travel, Accommodations, Expenses: Medac

Rosanna Parasole

Honoraria: Jazz Pharmaceuticals

Consulting or Advisory Role: Baxalta, Behring, Jazz Pharmaceuticals,

Takeda Pharmaceuticals

Travel, Accommodations, Expenses: Takeda Pharmaceuticals, CSL Behring

\section{Gudrun Göhring}

No relationship to disclose

Wolf-Dieter Ludwig

No relationship to disclose

Fiorina Casale

No relationship to disclose

Paul-Gerhardt Schlegel

No relationship to disclose

Giuseppe Basso

Honoraria; Becton Dickinson

Travel, Accommodations, Expenses: Jazz Pharmaceuticals

Valentino Conter

Honoraria: Jazz Pharmaceuticals

Travel, Accommodations, Expenses: Jazz Pharmaceuticals 


\section{Acknowledgment}

We thank the patients and families who participated in this trial, the physicians and nurses of all participating hospitals for their contribution to the completion of this study, and the study committees for productive discussions during the development and progress of the trial. We also thank the partners in the reference laboratories and all the technicians for their expert work in cytology, genetics, and MRD diagnostics and the data managers for their support in the study conduct. 